\title{
Outage behavior with delay and CSIT
}

\author{
Dinesh Rajan \\ Department of electrical engineering \\ Southern Methodist University, Dallas, Texas. \\ rajand@engr.smu.edu
}

\author{
Ashutosh Sabharwal and Behnaam Aazhang \\ Department of electrical and computer engineering \\ Rice University, Houston, Texas. \\ ashu@rice.edu, aaz@rice.edu
}

\begin{abstract}
In this paper, we demonstrate that the packet outage probability for fading channels can be significantly reduced by exploiting queuing delay and transmitter channel information. Queuing delay gain is conceptually similar to delay diversity, but at a packet time-scale instead of symbol time-scale. First, we compute a lower bound on outage probability assuming full channel state information at the transmitter (CSIT). We then construct simple outage minimizing transmission policies which adapt the rate and power of the transmitted signal based jointly on buffer occupancy and channel conditions. We demonstrate that the rate of decrease of outage with increasing transmitter channel information is higher for larger delays. We also address the closely coupled problem of designing a practical feedback channel which supplies the CSIT.
\end{abstract}

\section{INTRODUCTION}

In this paper, we propose a generalized framework for the design of transmission policies that reduce the probability of outage in block fading channels. Our emphasis is the interplay between queuing delay, amount of channel state information at the transmitter (CSIT) and outage probabilities. The proposed policies adapt the transmission rate and power to minimize the outage probability based on both the instantaneous buffer state and CSIT. Using the proposed scheme, we show that the outage probability reduces significantly (by an order of magnitude or more) for small increases in packet delays.

Communication through fading channels has been investigated under a variety of conditions (see [1] for a comprehensive review). The capacity of fading channels with channel state information (CSI) at the transmitter and receiver has been studied in [2]-[4]. Power control (PC) mechanisms have been widely investigated to improve performance in fading channels. With perfect CSIT and instantaneous (low delay) transmission of packets, ${ }^{1}$ the PC mechanism to minimize channel outage is given by "channel inversion" of the form, $P=\frac{e^{R}-1}{\gamma_{t}}$ when the instantaneous channel gain, $\gamma_{t}$, is greater than some threshold and zero otherwise. Thus, higher the instantaneous channel gain, lower the transmission power.

On the other hand, with infinite delay and buffering, the PC mechanism that achieves ergodic capacity, $C_{e}$, is given by the well known "water-filling" technique [5], $P_{t}=\frac{1}{\bar{\gamma}}-\frac{1}{\gamma_{t}}$ if $\gamma$ is greater than some threshold and zero otherwise. In this case, higher the instantaneous channel gain, higher the transmission power. The proposed adaptation schemes for finite delays are a combination of the two PC mechanisms discussed above;

\footnotetext{
${ }^{1}$ no packet can be queued for future transmission
}

the transmit power is a piecewise decreasing function of the instantaneous channel gain (see Fig. 1b).

Recently, there have been many works that adapt the system based jointly on the channel and traffic conditions. Transmit power minimization with increasing delays in Gaussian and fading channels has been investigated in [6]-[8]. The feedback of quantized CSI has been investigated in [9] with the objective of minimizing PC error caused by errors in feedback information using a loop filter at the transmitter. A combination of water-filling and channel inversion type power policy is proposed in [10] to minimize probability of service outage (as opposed to information outage).

In contrast to these, our work presented here is the first known work on investigating the interplay between outage probabilities, queuing delay and amount of CSIT in fading channels. Given $F$-bits of CSIT, we show that its utility is higher for larger delays. In other words, the rate of decrease of outage with increasing $F$ is larger for larger delays. We also compute a lower bound on outage assuming full CSIT and show that the amount of feedback required to achieve the outage lower bound increases with delay. We also give an empirical rule for choosing buffer size (equivalently delay) $L$ to 'best' utilize the $F$ bits of CSIT; choose $L$ to be the largest integer such that $F \geq \log _{2}(L(L+1) / 2+1)$. We also use a simple scalar quantizer of the instantaneous channel gain at the receiver to determine the feedback information. The results of this paper illustrate that similar outage performance can be achieved using various amounts of CSIT, delays and transmit powers. Thus the system designer has a lot of flexibility in choosing these quantities depending on the quality of service (QoS) requirements of the application.

Throughout the paper we assume constant rate packet arrivals. $^{2}$ The outage minimization problems in this paper are analytically intractable except in some simple cases and are solved using numerical techniques. We provide analytical results using Lagrangian techniques for small delays and conjecture some properties of the optimal solution in the general case, which are verified numerically. Also the receiver is assumed to have perfect instantaneous knowledge of CSI. We also assume packets are indivisible and have to be transmitted completely with in a time-slot; however, multiple packets may be transmitted in one time-slot.

The remainder of this paper is organized as follows. We

\footnotetext{
${ }^{2}$ Our results can be readily extended to time varying arrival traffic [11].
} 
introduce the notations used and formulate the problem in Section II. We compute a lower bound on outage probabilities in Section III. We propose outage reducing schemes with finite CSIT in Section IV. We conclude in Section V.

\section{PRoblem SetuP}

Consider a single user time-slotted system in which exactly one packet of fixed size $R$ arrives into a buffer of size $L$ packets, at the beginning of every time-slot. The number of packets in the queue at time $t$ is denoted by $q_{t}$. We use the convention that if a packet is transmitted in the same time-slot that it arrives, then the delay equals 1 . We assume a first come first serve policy and thus buffer size $L$ is assumed to be equal to the absolute delay bound, $D$.

We assume a block fading channel model in which the complex channel gain $h_{t}$ is constant over $T_{c}$ consecutive symbols, which is also the length of one time-slot. The channel fading $h_{t}$ is assumed to vary independently from one time-slot to another. The transmitted signal $\mathbf{x}_{t}$ depends on the number of packets transmitted at time $t$ and on the coding and modulation scheme used. The complex received signal $\mathbf{y}_{t}$ is given by,

$$
\mathbf{y}_{t}=h_{t} \mathbf{x}_{t}+\mathbf{z}_{t},
$$

where $\mathbf{z}_{t}$ is the additive noise. The transmitted signal $\mathbf{x}_{t}$, the received signal $\mathbf{y}_{t}$ and the additive noise $\mathbf{z}_{t}$ are $T_{c}$ dimensional complex vectors (vector quantities are represented in boldface). The real and imaginary parts of $h_{t}$ are assumed to be independent zero mean Gaussian, each with variance $1 / 2$. Also, the additive noise $\mathbf{z}_{t}$ is assumed to be circularly symmetric Gaussian with zero mean and covariance $\sigma^{2} \mathbf{I}_{T_{c}}$ where $\mathbf{I}_{T_{c}}$ is the identity matrix of size $T_{c}$.

For large $T_{c}$, the conditional mutual information between $\mathbf{x}_{t}$ and $\mathbf{y}_{t}, I\left(\mathbf{y}_{t} ; \mathbf{x}_{t} \mid h_{t}\right)$, is a good indicator of the performance of practical codes [12]. This mutual information is given by

$$
I\left(\mathbf{y}_{t} ; \mathbf{x}_{t} \mid h_{t}\right)=T_{c} \log \left(1+\frac{P_{t}\left|h_{t}\right|^{2}}{\sigma^{2}}\right)=T_{c} \log \left(1+P_{t} \gamma_{t}\right),
$$

where $\gamma_{t}=\frac{\left|h_{t}\right|^{2}}{\sigma^{2}}$ is the normalized channel gain and $P_{t}$ is the transmit power during time-slot $t$.

The total packet loss probability, $\Pi$, depends on the probability of buffer overflows, $\Pi_{b}$, and the frame error rate of the actual transmission scheme. In this paper, we use the probability of outage in the channel given by $\Pi_{o}=$ $\operatorname{Pr}\left\{I\left(\mathbf{y}_{t} ; \mathbf{x}_{t} \mid h_{t}\right)<R\right\}$, as an indicator of the frame error rate ${ }^{3}$ in practical systems [12]. Hence, $\Pi=\Pi_{b}+\left(1-\Pi_{b}\right) \Pi_{o}$.

The transmit power $P_{t}$ during a time-slot depends on two factors: the number of packets $q_{t}$ available for transmission and $\hat{\gamma}_{t}$ the estimate at the transmitter of the normalized channel gain $\gamma_{t}$ : Thus, $P_{t}=f\left(q_{t}, \hat{\gamma}_{t}\right) .{ }^{4}$ Note that once $P_{t}$ is computed, the instantaneous transmission rate is also determined. In this paper, we consider a generalized class of thresholding functions $f$ which are described later in this section.

\footnotetext{
${ }^{3}$ By using the information theoretically defined $\Pi_{o}$, we abstract away from the actual coding scheme used.

${ }^{4}$ In this paper we assume that $\hat{\gamma}_{t}$ is an error free quantized version of $\gamma_{t}$. The channel estimation error and errors in the feedback channel are ignored.
}

The optimization problem we consider can be formally stated as follows,

$$
\begin{aligned}
& \min _{f} \Pi \text {, } \\
& \mathbb{E}\left[P_{t}\right] \leq P_{0}
\end{aligned}
$$

where $P_{0}$ is the average power constraint. The optimization problem (2) is solved for a given delay bound $D$. First, we compute a lower bound for outage probability by considering $\hat{\gamma}_{t}=\gamma_{t}$, i.e., by assuming full CSIT. Then, we consider $\hat{\gamma}_{t}$ to be a $F$-bit quantization of $\gamma_{t}$ and construct outage minimizing policies that vary the transmission power and rate. Finally, when there is no CSIT, $\hat{\gamma}_{t}=\mathbb{E}\left[\gamma_{t}\right]$ : The transmission power is then a constant and that case is treated in [13]. Next, we define the class of functions $f$ considered in solving (2).

\section{A. Special class of functions}

Solving (2) over the set of all feasible functions $f$ is a hard (and open) problem. In this paper, we consider a generalized class of functions that determine the instantaneous transmission rate and power. The class of functions $f$ considered involve the selection of thresholds $\gamma_{k, l}, 1 \leq l \leq k \leq L$ such that during time-slot $t, l$ packets are transmitted if $q_{t}=k$ and $\gamma_{k, l} \leq \hat{\gamma}_{t}<\gamma_{k, l+1}$, i.e., if buffer has $k$ packets and channel gain lies between certain thresholds. No packets are transmitted if buffer state $q_{t}=k$ and channel gain $\gamma_{t}<\gamma_{k, 1}$. For notational simplicity, we let $\gamma_{k, 0}=0, \gamma_{k, k+1}=\infty, \forall k$. There is also a natural constraint imposed on the thresholds $\gamma_{k, l}$ namely $\gamma_{k, l} \leq \gamma_{k, m}$ if $l \leq m$, i.e., more packets are transmitted when the instantaneous channel gain $\gamma_{t}$ is higher. The thresholds $\left\{\gamma_{k, l}\right\}$ (illustrated in Fig. 1 for buffer state $L$ ) are also the boundary regions of a scalar quantizer of $\gamma_{t}$ at the receiver. We consider transmission schemes (determined by $f$ )

\section{Partial CSIT}

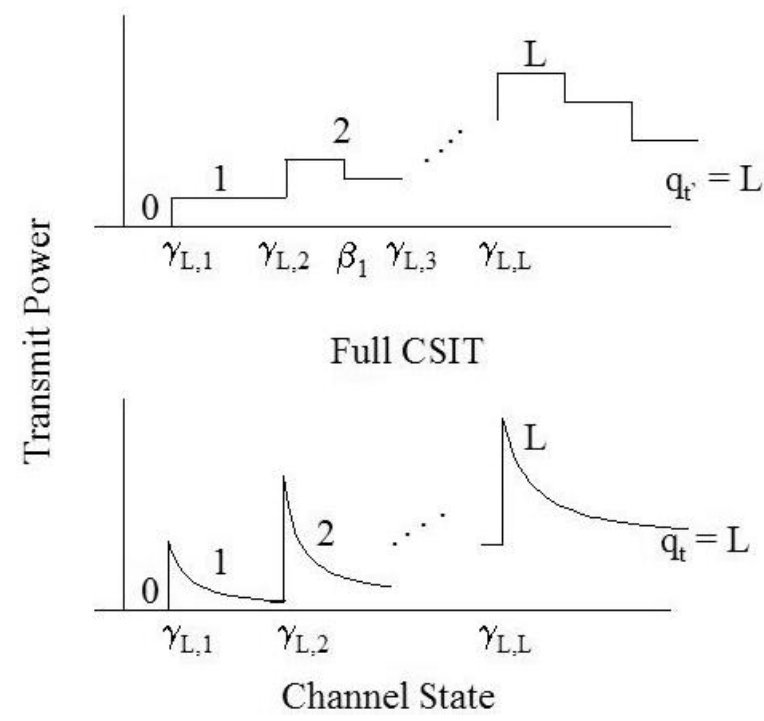

Fig. 1. Rate and power control policy with partial or full CSIT. The number of packets transmitted in each case is also indicated. 
in which outage does not occur in the channel, but only due to packet dropping arising from buffer overflows. Hence, $\Pi_{o}=0$ and $\Pi=\Pi_{b}{ }^{5}$ Zero outage in the channel can be ensured by choosing enough power to ensure that the instantaneous mutual information is greater than $R$ (see (7) and (11)). ${ }^{6}$ Since we assume that exactly one packet arrives during every timeslot, buffer overflows only occurs in the $q_{t}=L$ buffer state. ${ }^{7}$ The queue state $q_{t}$ forms a stationary Markov chain with $L$ states. The transition probabilities $p_{j i}$ between the different queue states defined as $p_{j i}=\operatorname{Pr}\left\{q_{t+1}=j \mid q_{t}=i\right\}$, can be computed as

$$
p_{j i}= \begin{cases}e^{-\gamma_{i, 1}}-e^{-\gamma_{i, 2}} & \text { if } i=j \neq K \\ 1-e^{-\gamma_{i, 1}} & \text { if } j=i+1 \\ 1-e^{-\gamma_{K, 2}} & \text { if } i=j=K \\ e^{-\gamma_{i, i-j+1}}-e^{-\gamma_{i, i-j+2}} & \text { if } j<i \\ 0 & \text { else }\end{cases}
$$

The stationary probability of being in buffer state $q_{t}=i$, denoted by $s_{i}$ (which is also the invariant distribution of the Markov chain) is then given by,

$$
\mathrm{Cs}=\mathbf{s}
$$

where $\mathbf{s}=\left[s_{1} s_{2} \ldots s_{L}\right]^{\prime}$ and $\mathbf{C}$ is an $L \times L$ matrix whose $i^{t h}$ row and $j^{t h}$ column is $p_{i j}$. Thus, the packet loss probability, which depends on the thresholds $\gamma_{i, j}$, is given by

$$
\Pi=\Pi_{b}=s_{L} \int_{0}^{\gamma_{L, 1}} e^{-\gamma} d \gamma=s_{L}\left(1-e^{-\gamma_{L, 1}}\right) .
$$

We now proceed to find a lower bound on outage probabilities assuming full CSIT.

\section{OUTAGE MINIMIZATION - LOWER BOUND}

Delay bound $D=2$ : To gain a better understanding of the tradeoffs between $\Pi$ and $D$, we first solve (2) for the special case of $D=2 \equiv L=2$ packets. Now, the stationary probabilities of being in queue states $q_{t}=1$ and $q_{t}=2$ can be computed in closed form by solving (4) and are given by,

$$
s_{1}=\frac{1-e^{-\gamma_{1,1}}}{1-e^{-\gamma_{1,1}}+e^{-\gamma_{2,2}}} ; s_{2}=\frac{e^{-\gamma_{2,2}}}{1-e^{-\gamma_{1,1}}+e^{-\gamma_{2,2}}}
$$

Recall that $l$ packets are transmitted when $q_{t}=k$ and $\gamma_{k, l} \leq$ $\hat{\gamma}_{t} \leq \gamma_{k, l+1}$. The transmission power, $P_{t}$, which ensures zero outage in the channel is given by

$$
P_{t}=f\left(k, \hat{\gamma}_{t}\right)=\frac{e^{l R}-1}{\hat{\gamma}_{t}} \text { if } \gamma_{k, l} \leq \hat{\gamma}_{t} \leq \gamma_{k, l+1}
$$

Consequently, the total transmission power is given by $\mathbb{E}\left[P_{t}\right]=$ $\left(e^{R}-1\right)\left(s_{1} \operatorname{Ei}\left(\gamma_{1,1}\right)+s_{2} \operatorname{Ei}\left(\gamma_{2,1}\right)-\operatorname{Ei}\left(\gamma_{2,2}\right)\right)+s_{2}\left(e^{2 R}-\right.$ 1) $E i\left(\gamma_{2,2}\right)$, where $E i(x)=\int_{x}^{\infty} \frac{e^{-\gamma}}{\gamma} d \gamma$. The outage is given

\footnotetext{
${ }^{5}$ In this paper, we use outage or total packet loss probability to refer to $\Pi$ and channel outage to refer to $\Pi_{o}$.

${ }^{6}$ Note that with no CSIT, zero outage in the channel can not be guaranteed.

${ }^{7}$ Also, the probability of buffer being empty is zero.
}

by $\Pi_{2}=s_{2} \int_{0}^{\gamma_{2,1}} e^{-\gamma} d \gamma=s_{2}\left(1-e^{-\gamma_{2,1}}\right)$, where subscript 2 in $\Pi_{2}$ indicates that $D$ equals 2 . Thus, (2) can be rewritten as,

$$
\Pi_{2}^{*}=\min _{\substack{\gamma_{1,1}, \gamma_{2,1}, \gamma_{2,2} \\ \mathbb{E}\left[P_{t}\right]<P_{0}}} s_{2}\left(1-e^{-\gamma_{2,1}}\right) .
$$

Proposition 1: If $\gamma_{2,2}^{*}$ and $\gamma_{1,1}^{*}$ are solution to (8), then $\gamma_{2,2}^{*}=\gamma_{1,1}^{*} e^{R}$

Proof: The result follows from Lagrangian optimization techniques and is omitted here in the interest of space.

Using Lagrangian techniques to solve (8) also results in the following closed form relationship,

$$
\begin{aligned}
\gamma_{2,1}^{*}= & E i^{-1}\left[\left(1+\frac{e^{-\gamma_{1,1}^{*} e^{R}}}{1-e^{-\gamma_{1,1}^{*}}}\right) \frac{\bar{P}}{\left(e^{R}-1\right)}-\right. \\
& \left.\frac{e^{-\gamma_{1,1}^{*} e^{R}}}{1-e^{-\gamma_{1,1}^{*}}} \operatorname{Ei}\left(\gamma_{1,1}^{*}\right)-e^{R} \operatorname{Ei}\left(\gamma_{1,1}^{*} e^{R}\right)\right] .
\end{aligned}
$$

Finding the optimal thresholds completely in closed form is intractable and hence we use numerical optimization techniques. Arbitrary delays: An approach similar to the $D=2$ case can be used to compute the lower bound for larger delays. In this case, the optimization problem is

$$
\Pi_{L}^{*}=\underset{P_{\text {full } C S I T}=\sum_{k=1}^{L} \sum_{l=1}^{k} s_{k}\left(e^{l R}-1\right)\left(E i\left(\gamma_{k}, l\right)-E i\left(\gamma_{k}, l+1\right)\right)<P_{0}}{\min _{i, j}} \quad s_{L}\left(1-e^{-\gamma_{L, 1}}\right) .
$$

Based on Proposition 1, we conjecture that the optimal thresholds follow the relations

$$
\gamma_{i, i}^{*}=e^{(i-1) R} \gamma_{1,1}^{*}, i=2,3, \ldots, L .
$$

Although proving (10) analytically is intractable, we found it to hold in all our simulations. A consequence of (10) is that the power required to transmit $k$ packets when $q_{t}=k$ is no greater than $\frac{e^{k R}-1}{e^{(k-1) R} \gamma_{1,1}} \approx \frac{e^{R}-1}{\gamma_{1,1}}$, which is the power needed to transmit 1 packet when $q_{t}=1$. In other words, by increasing the delay and waiting for buffer to build up to $k$ packets, we transmit $k$ packets with the same power as that needed to transmit one packet in buffer state 1 . We solve (9) using numerical optimization and a typical solution is given below.

Example 1: Let $L=4, R=0.8$ and $P_{0}=1$. The optimal thresholds $\left\{\gamma_{k, l}\right\}$ are given by $\gamma_{1,1}^{*}=0.6762, \gamma_{2,1}^{*}=$ $0.5859, \gamma_{2,2}^{*}=1.5067, \gamma_{3,1}^{*}=0.5367, \gamma_{3,2}^{*}=1.3043, \gamma_{3,3}^{*}=$ $3.352, \gamma_{4,1}^{*}=0.4998, \gamma_{4,2}^{*}=1.1945, \gamma_{4,3}^{*}=2.8999, \gamma_{4,4}^{*}=$ 7.4476 Clearly, $\log \left(\frac{\gamma_{2,2}^{*}}{\gamma_{1,1}^{*}}\right)=0.801, \log \left(\frac{\gamma_{3,3}^{*}}{\gamma_{1,1}^{*}}\right)=$ $1.601, \log \left(\frac{\gamma_{4,4}^{*}}{\gamma_{1,1}^{*}}\right)=2.399$ thus strengthening conjecture (10). Further note that conditioned on $q_{t}$, the power $P_{t}$ is a piecewise decreasing function of $\gamma_{t}$ (see Fig. 1). For $\gamma_{k, l} \leq \gamma_{t}<\gamma_{k, l+1}$, the power decreases with $\gamma_{t}$. At each of the thresholds $\gamma_{k, l}$, there is a discontinuity, and the power increases to transmit one additional packet.

The plot of the lower bound on $\Pi$ versus delay $D$ is given in Fig. 2 for two different values of SNR. The arrival rate equals 0.9 nats/sec/Hz. At low SNR, the ergodic capacity $C_{e}$ of the 
channel equals 0.7 nats $/ \mathrm{sec} / \mathrm{Hz}$ which is lower than the average arrival rate. Consequently, the lower bound on packet outage probability does not approach zero with increasing delays: The lower bound asymptotically equals $1-\frac{C_{e}}{R}=\frac{2}{9}$. At high $\mathrm{SNR}$, the ergodic capacity equals 1 nats $/ \mathrm{sec} / \mathrm{Hz}$ which is larger than the average arrival rate and the lower bound on outage decreases rapidly with increasing delays and approaches zero. The variation of lower bound with SNR for three different delays is given in Fig. 4. Clearly, with increasing SNR, the lower bound reduces for all delays. For a fixed SNR, the reduction in the lower bound as $D$ increases from 1 to 3 is substantial.

\section{OUtAGE PROBABILITIES With PARTIAL CSIT}

In Section III we found lower bounds on outage probability assuming perfect CSIT. In this section, we propose thresholding type power and rate control mechanisms that uses $F$ feedback bits to reduce the outage for various delays.

In the thresholding scheme introduced in Section II-A, the number of thresholds in buffer state $i$ equals $i$ and hence the total number of thresholds $N=\sum_{i=1}^{L} i=\frac{L(L+1)}{2}$. These $N$ thresholds partition $\mathcal{R}^{+}$(which is the range of $\gamma_{t}$ ) into $N+1$ regions and hence we need $\log _{2}(N+1)$ bits to signal the region in which the instantaneous channel gain lies.

Initially assume that $F \geq \log _{2}(N+1)$. We now consider a modified version of the thresholding scheme introduced in Section II-A. In addition to the different $\gamma_{i, j}$ thresholding parameters, we introduce parameters $\beta_{1}, \beta_{2}, \ldots, \beta_{m}$, where $m=2^{F}-N-1$. The $\left\{\gamma_{i, j}\right\}$ and $\left\{\beta_{i}\right\}$ parameters partition $\mathcal{R}^{+}$into $2^{F}$ regions. While the $\gamma_{i, j}$ parameters determine the number of packets transmitted, the $\beta$ parameters determine the transmit power. It is assumed that both the transmitter and receiver know these thresholding parameters a priori. Thus $\hat{\gamma}_{t}$ is an $F$-bit scalar quantized value of $\gamma_{t}$ where the quantizer thresholds are chosen to minimize the outage probability. The receiver transmits a $F$-bit code word corresponding to the region to which $\gamma_{t}$ belongs.

As before, $l$ packets are transmitted if $q_{t}=k$ and $\gamma_{k, l} \leq$ $\gamma_{t}<\gamma_{k, l+1}$ using power $P_{t}$ given by

$$
P_{t}=f\left(k, \hat{\gamma}_{t}\right)=\frac{e^{l R}-1}{\beta} \text { if } \gamma_{k, l} \leq \hat{\gamma}_{t}<\gamma_{k, l+1},
$$

where $\beta$ is the largest threshold that lies between $\gamma_{k, l}$ and $\hat{\gamma}_{t}$ and is given by

$$
\beta=\underset{\substack{\left\{\beta_{1}, \beta_{2}, \ldots, \beta_{m}, \gamma_{k, l}\right\} \\ x<\hat{\gamma}_{t}}}{\arg \max } x
$$

The transmit power is now a step function (Fig. 1). In the special case of $F=\log _{2}(N+1) \Rightarrow m=0$, the transmit power is given by $\frac{e^{l R}-1}{\gamma_{k, l}}$. Asymptotically, as $F \rightarrow \infty$, this scheme approaches the adaptation mechanism for full CSIT (Section III) and therefore the outage lower bound is achieved. The total transmit power, $P_{\text {partial CSIT }}$, can be computed as,

$$
P_{\text {partial CSIT }}=\sum_{k=1}^{L} s_{k} \sum_{l=1}^{k} \int_{\gamma_{k, l}}^{\gamma_{k, l+1}} \frac{\left(e^{l R}-1\right)}{\beta} e^{-\gamma} d \gamma
$$

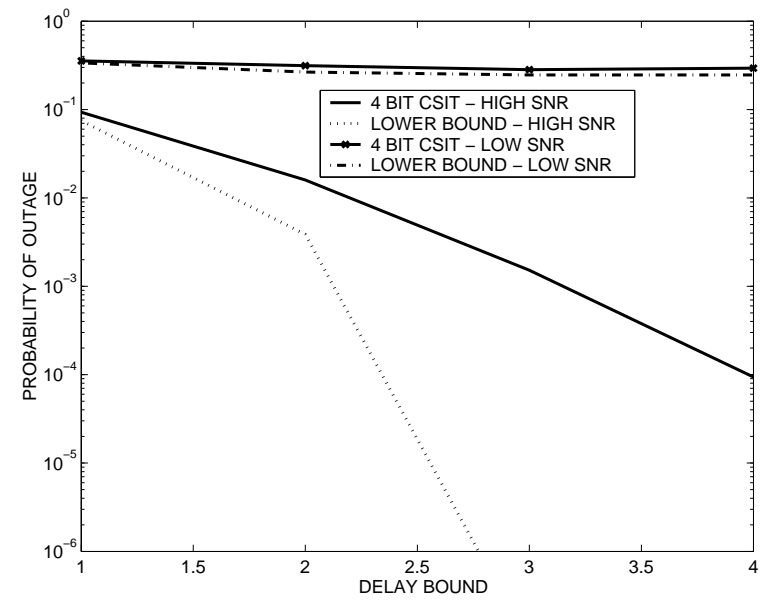

Fig. 2. Outage probabililty versus delay for 4 bit CSIT.

In this case, the outage minimization problem is similar to (9) with $P_{\text {full CSIT }}$ replaced by $P_{\text {partial CSIT }}$, which we solve using numerical optimization techniques. We study the variation of outage with regard to 3 different system parameters: delay $D$, number of feedback bits $F$ and SNR by keeping the other parameters constant.

Outage versus delay: The variation of outage versus delay is given in Fig. 2 for two different values of SNR. We see that for low SNR the outage lower bound is achieved at all delays. For high SNR, the outage decreases at a slower rate with finite amount of CSIT than the lower bound. Thus the difference between the lower bound and outage with 4-bits of $F$ increases with $D$. We see that at high SNR, as $D$ increases from 1 to 3 , the outage probability reduces by nearly 2 orders of magnitude.

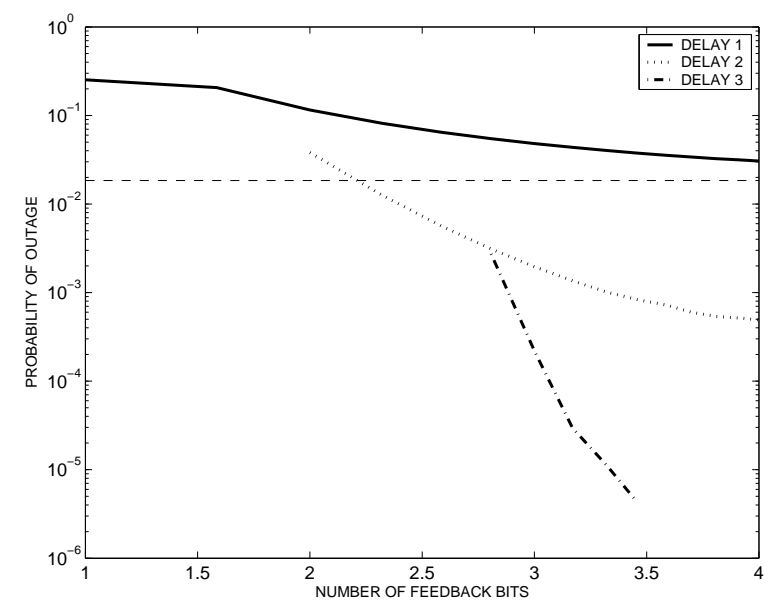

Fig. 3. Outage probabilities versus number of feedback bits. The horizontal lines indicate the lower bound on outage probabilities.

Outage versus number of feedback bits: The plot of outage probabilities versus $F$ is given in Fig. 3 for $D=1$. The loss probability with 0 feedback bits is the outage probability with 


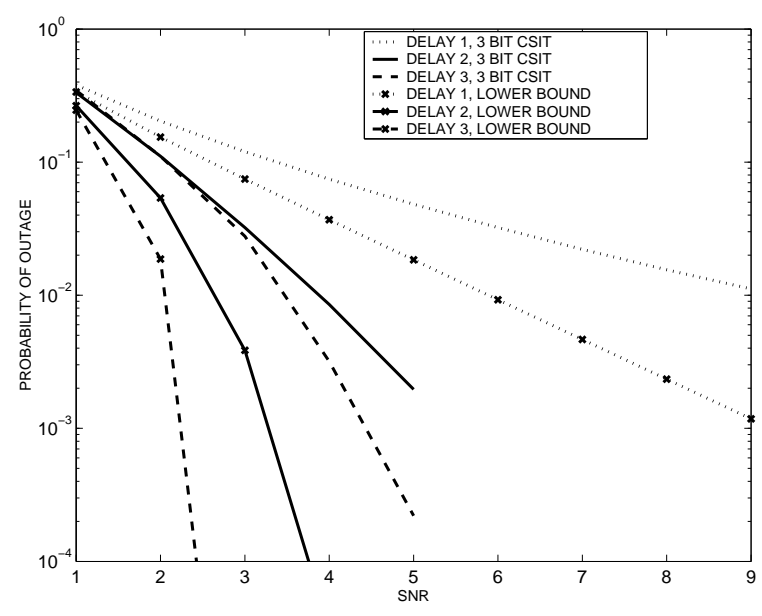

Fig. 4. Outage probabilities versus SNR for various delays using 3 bit CSIT.

no CSIT. We find that with 1 bit of feedback, the outage probability does not reduce much from the no CSIT case. However, as the feedback increases beyond 1 bit, the outage probability reduces drastically. Asymptotically, as $F$ increases, the outage probability approaches the lower bound.

The reason why one bit of feedback does not reduce outage much is as follows. With no CSIT, the optimal PC policy transmits at constant power $P_{0} \forall \gamma_{t}$. With one bit CSIT, the PC policy we consider is of the form,

$$
P_{t}= \begin{cases}\frac{\left(e^{R}-1\right)}{\gamma_{1,1}} & \text { if } \gamma_{t}>\gamma_{1,1} \\ 0 & \text { else }\end{cases}
$$

where $\gamma_{1,1}$ is chosen to satisfy the average power constraint, $\int_{\gamma_{1,1}}^{\infty} \frac{\left(e^{R}-1\right)}{\gamma_{1,1}} e^{-\gamma} d \gamma \leq P_{0}$. As the SNR increases, $\gamma_{1,1}$ decreases and (13) is almost equivalent to transmitting at constant power $P_{0} \forall \gamma_{t}$. Hence, there is no significant reduction in outage with 1 bit CSIT. ${ }^{8}$

We also plot the variation of outage with $F$ for delays 2 and 3 in Fig. 3.Again, note that as $F$ increases, the outage decreases and eventually reaches the lower bound. However, larger the delay $D$, higher the number of feedback bits $F$ needed to achieve the lower bound. ${ }^{9}$ We find that the rate of decrease of outage with $F$ is larger for larger delays. Hence, for given $F$, to best utilize the partial CSIT, the delay should be chosen as the largest $D^{\prime}\left(\equiv L^{\prime}\right)$ such that $\log _{2}\left(\frac{D^{\prime}\left(D^{\prime}+1\right)}{2}+1\right) \leq$ $F$, subject to not violating the delay bound.

Outage versus SNR: The plot of outage versus SNR using $F=3$ bits is given for three different delays in Fig. 4. For comparison, the outage lower bounds for each of the three buffer lengths is shown. We see that the rate of decrease of outage with SNR is larger for larger $D$, similar to the variation of the lower bound. The savings in power for small increases in $D$ is substantial. For example, at an outage of $10^{-2}$ increasing $D$ from 1 to 3 , results in more than $5 \mathrm{~dB}$ savings in power.

\footnotetext{
${ }^{8} \mathrm{PC}$ policies in which $P_{t} \neq 0 \forall \gamma_{t}$ also resulted in similar outage values.

${ }^{9}$ The lower bound for $D=2,3$ lies outside the scale of the figure and is not shown.
}

Most of the power savings comes from the initial increase in delays. The power savings by increasing delays further only results in diminishing returns. An important implication of our result is that small increases in delay leads to large reductions in power at any given outage probability.

So far, we have proposed outage minimizing techniques when $F \geq \log _{2}(N+1)$. If $F<\log _{2}(N+1)$ then one can still use the thresholding scheme of Section II-A, but instead of allowing the buffer to build up to $L$ packets, we allow the buffer only to build up to the largest $\tilde{L}$ such that $F \geq \log _{2}\left(\frac{\tilde{L}(\tilde{L}+1)}{2}+1\right)$. Clearly, such a scheme does not utilize the available buffer optimally and is only an upper bound on achievable performance. If $F$ equals 0 , then we have no CSIT and outage reduction is possible by increasing encoding delay:see [13] for preliminary results.

\section{Conclusions}

In this paper, we showed that outage probabilities in fading channels can be reduced by exploiting a combination of queuing delay and CSIT. We introduced simple thresholding type power control mechanisms that significantly reduce outage probabilities for small increases in delay. The effects of imperfections in the channel estimate at the receiver and in the feedback channel on outage performance needs to be investigated in future work.

\section{REFERENCES}

[1] E. Biglieri, J. G. Proakis, and S. Shamai, "Fading channels: Informationtheoretic and communications aspects," IEEE Trans. Inf. Thy., vol. 44, no. 6, pp. 2619-2692, October 1998.

[2] M. Medard, "The effect upon channel capacity in wireless communications of perfect and imperfect knowledge of the channel," IEEE Trans. Inf. Thy., vol. 46, no. 3, pp. 935-946, May 2000.

[3] M. Medard and A. Goldsmith, "Capacity of time varying channels with side information at the sender and receiver," in Proc. ICC, Vancouver, Canada, June 1999.

[4] A. J. Goldsmith and P. P. Varaiya, "Capacity of fading channels with channel side information," IEEE Trans. Inf. Thy., vol. 43, no. 6, pp. 1986-1992, Nov. 1997.

[5] T. M. Cover and J. A. Thomas, Elements of Information Theory. Wiley, New York, 1991.

[6] B. Collins and R. L. Cruz, "Transmission policies for time varying channels with average delay constraints," in Proc. Allerton Conf. on Comm. Control and Computing., Monticello, IL, 1999, pp. 709-717.

[7] R. A. Berry and R. G. Gallager, "Communication over fading channels with delay constraints," IEEE Trans. Inf. Thy., vol. 48, no. 5, pp. 11351149, May 2002.

[8] D. Rajan, A. Sabharwal, and B. Aazhang, "Delay bounded packet scheduling of bursty traffic over wireless channels," IEEE Trans. Inf. Thy., vol. 50, no. 1, pp. 125-144, January 2004.

[9] H.-J. Su and E. Geraniotis, "Adaptive closed loop power control with quantized feedback and loop filtering," IEEE Trans W. Comm., vol. 1, no. 1, pp. 76-87, January 2002.

[10] J. Luo, L. Lin, R. Yates, and P. Spasojevic, "Service outage based power and rate allocation," in Proc. Conf. on Inf. Sciences and Systems., Baltimore, MD, March 2001.

[11] A. Sabharwal, D. Rajan, and B. Aazhang, "Dual problems in power control," in Proc. Allerton Conf. on Comm. Control and Computing., Monticello, IL, October 2001.

[12] L. H. Ozarow, S. Shamai, and A. D. Wyner, "Information theoretic considerations for cellular mobile radio," IEEE Trans. Veh. Tech., vol. 43, no. 2, pp. 359-378, May 1994.

[13] D. Rajan, "Power efficient transmission policies for multimedia traffic over wireless channels," Ph.D. dissertation, Rice University, April 2002. 